\title{
Clinical Pathological Analysis in 104 Ductal Breast Cancer Cases
}

\author{
Yueling Jin', Xiuying Huang², Yanfei Li' ${ }^{1}$ Xuemei Li' ${ }^{1}$, Zhensheng Dai ${ }^{3}$ \\ ${ }^{1}$ Department of Pathology, Shanghai University of Medicine \& Health Sciences, Shanghai, China \\ ${ }^{2}$ Departments of Emergency, Shanghai Pudong Hospital Affiliated to Fudan University, Shanghai, China \\ ${ }^{3}$ Department of Hematology-Oncology, Shanghai Pudong Hospital Affiliated to Fudan University, Shanghai, China \\ Email: *zhenshengdai@126.com
}

How to cite this paper: Jin, Y.L., Huang, X.Y., Li, Y.F., Li, X.M. and Dai, Z.S. (2017) Clinical Pathological Analysis in 104 Ductal Breast Cancer Cases. Journal of Biosciences and Medicines, 5, 37-43.

https://doi.org/10.4236/jbm.2017.512005

Received: July 16, 2017

Accepted: November 18, 2017

Published: November 21, 2017

\begin{abstract}
Ductal breast cancer is a clinically heterogeneous disease; we investigate the correlation between tumor size, comedo necrosis, number of cell mitoses and axillary lymph node metastasis in ductal breast cancer. In this study, 104 ductal breast cancer specimens were collected and divided into 3 groups: T1 group (tumor size $\leq 2 \mathrm{~cm}$ ), T2 group $(2<$ tumor size $\leq 5 \mathrm{~cm}$ ) and T3 group (tumor size $>5.0 \mathrm{~cm}$ ). Among those specimens, 95 cases were diagnosed with invasive ducted carcinoma, and 9 cases were ductal carcinoma in-situ (DCIS). Results show that $\mathrm{T} 3$ group has a higher rate of axillary lymph node metastasis than T2 group and T1 group; T2 group has a higher rate of lymph node metastasis than T1 group. The patients with the number of cell mitoses $(\geq 10)$ were also has a higher rate of axillary lymph node metastasis $(P=0.0139)$ than the patients with the number of cell mitoses $(<10)$. No significance was found between comedo necrosis lesions and axillary lymph node metastasis, though the frequency of comedo necrosis lesions in patients with axillary lymph node metastasis was higher than those in non-metastatic patients. It is concluded that the tumor size and the number of cell mitoses were risk factors for axillary lymph node involvement in ductal breast cancer.
\end{abstract}

\section{Keywords}

Pathological Analysis, Ductal Breast Cancer, Lymph Node Metastasis

\section{Introduction}

Breast cancer is the most common malignant tumor among women, most of which are confirmed by pathological examination. In this study, 104 cases of ductal breast cancer specimens were collected. We analyzed and discussed the 
relationship between the tumor size, comedo necrosis, the number of cell mitoses and axillary lymph node metastasis.

\section{Patients}

\subsection{Patient Samples}

Samples were retrieved from 104 ductal breast cancer patients, tissues were made into paraffin blocks and HE staining, diagnosed by pathological doctors. Pathological type: 9 cases of carcinoma in situ (Figure 1(a)), 95 cases of invasive ductal carcinoma (Figure 1(b)).

\subsection{Age Distribution of Patients}

All cases were female. Age ranged from 24 to 83 years old. 2 cases of breast cancer patients under the age of 30,10 cases of 31 to 40 years, 31 cases of 41 to 50 years, 20 cases of 51 to 60 years, 22 cases of 61 to 70 years, 19 cases of over 71 years old (Table 1); 56 tumors are in left breast, 47 cases in right, 1 case was bilateral.

\section{Methods}

\subsection{Hematoxylin and Eosin Staining}

The original tumours have been fixed in $4 \%$ formaldehyde for $24 \mathrm{~h}$, and then embedded in paraffin. Paraffin blocks were made of 3 - $4 \mu \mathrm{m}$ thick sections, after routine hematoxylin and eosin staining; the pathologist observed the histopathological changes and classified the grade under optical microscope.

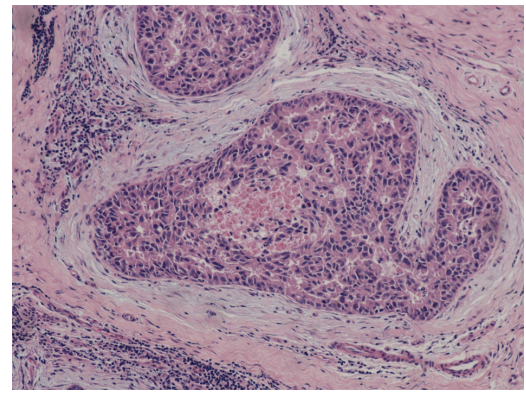

(a)

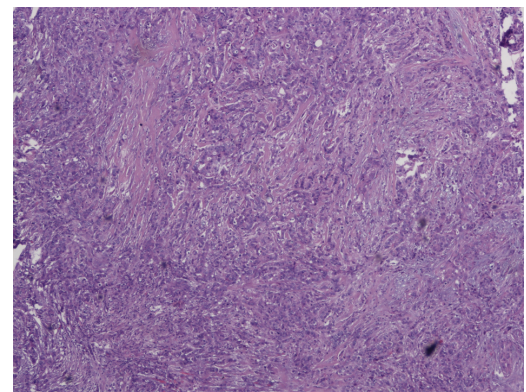

(b)

Figure 1. (a) Pathological type: 9 cases of carcinoma in situ; (b) 95 cases of invasive ductal carcinoma.

Table 1. The relationship between tumor size and lymph node metastasis.

\begin{tabular}{cccc}
\hline Tumor size & number of patient (cases) & $\begin{array}{c}\text { lymph node }(+) \\
\text { (cases/percentage) }\end{array}$ & $\begin{array}{c}\text { lymph node }(-) \\
\text { (cases/percentage) }\end{array}$ \\
\hline T1 & 35 & $9(25.7 \%)$ & $24(74.3 \%)$ \\
T2 & 56 & $29(51.8 \%)$ & $28(48.2 \%)$ \\
T3 & 13 & $12(92.3 \%)$ & $2(7.7 \%)$ \\
\hline
\end{tabular}

Lymph node positive rate of group T2 compared with group T1, $P=0.0418, P<0.05$; T3 compared with T2, $P=0.0184, P<0.05$; T3 compared with T1, $P=0.0003, P<0.05$. 


\subsection{Classification of Samples}

The largest diameter size of gross tumor as the measure standards, the samples were divided into three groups: T1 group (tumor size $\leq 2 \mathrm{~cm}$ ), T2 group $(2<$ tumor size $\leq 5 \mathrm{~cm}$ ) and $\mathrm{T} 3$ group (tumor size $>5.0 \mathrm{~cm}$ ). According to Bloom-Richardson histological grading system, we divided infiltrative breast cancer into I, II, and III grade.

\subsection{Statistical Analyses}

Analysis was performed using GraphPad software, statistical analysis was performed by Fisher's exact test, $P<0.05$ was considered to indicate a statistically significant difference.

\section{Results}

Relationship between breast cancer tumor size and axillary lymph node metastasis. Grossly, T1 group 35 cases, T2 group 56 cases, and T3 group 13 cases. Results shown: $\mathrm{T} 3$ group has a higher rate of axillary lymph node metastasis (92.3\%) than T2 group (51.8\%) and T1 group (25.7\%); T2 group (51.8\%) has a higher rate of lymph node metastasis than $\mathrm{T} 1$ group (25.7\%). All the comparison between those two groups were statistical significant $(\mathrm{P}<0.05)($ Table 1$)$.

The relationship between comedo necrosis, lymph node metastasis and stage of breast cancer. In the 50 breast cancer cases who had lymph node metastasis, there were 39 cases accompanied comedo necrosis, while 21 cases with comedo necrosis among 53 cases with no lymph node metastasis. Although the rate of lymph node metastasis patients accompanied comedo necrosis (39/103) was higher than the rate of without lymph node metastatic rate (21/103), there was no significant difference $(P>0.05)$. Results also shown that, with the increase of tumor clinical grade, the number of samples with comedo necrosis also gradually increased, but there was no significant difference (Table 2).

The relationship between the number of cell mitoses and axillary lymph node metastasis. Results are shown in table 3. In the high-power fields $(\times 400)$, we count the number of cell mitoses. 104 cases of resected specimens with invasive breast cancer were divided into two groups: 0 - 9 mitoses in high-power fields as a group I, $\geq 10$ mitoses as a group II. We analyzed the difference between those

Table 2. The relationship between comedo necrosism lymph node metastasis and stage of breast cancer.

\begin{tabular}{cccc}
\hline grade & $\mathrm{n}$ & comedo necrosis/lymph node $(+)$ & comedo necrosis/lymph node $(-)$ \\
\hline I & 15 & $2(13.3 \%)$ & $3(20.0 \%)$ \\
II & 29 & $10(34.5 \%)$ & $7(24.1 \%)$ \\
III & 59 & $27(45.8 \%)$ & $11(18.6 \%)$ \\
uncertain & 1 & & \\
\hline
\end{tabular}

n: number of cases. Group comedo necrosis/lymph node (+) compared with group comedo necrosis/lymph node (+), $P>0.05)$ 
two groups. The results show that the number of patients with axillary lymph node metastasis in group II is more than that of group I. The difference was statistically significant $(P<0.05)$.

\section{Discussion}

Ductal breast cancer is a clinically heterogeneous disease. About $10 \%$ to $15 \%$ of breast cancer patients have distant metastases within 2 years, which significantly reduced the 5 year survival rate [1]. Staging, Tumor size and lymph node metastasis are important prognostic factors for breast cancer metastasis, and in those factors, lymph node metastasis is the most important effect on ductal breast cancer prognosis.

We analyzed the pathological diagnoses on 104 cases of ductal breast cancer. The results were shown in Figure 2. The peak of the age on breast cancer is still more than 40 years old. But it is worthy of mentioning that within 12 patients less than 40 years old, 7 (58.33\%) had axillary lymph node metastases.

The youngest was 24 years old, who was diagnosed as invasive ductal carcinoma, axillary lymph node metastasis is positive. Therefore, it is suggested that in any age group of women, as long as the breast tumor is found, it should be pay great attention to do a physical check, so to improve the early diagnosis rate, and do the best measures on improving the survival of breast cancer.

Table 1 shows the relationship between the breast cancer tumor size and axillary lymph node metastasis, the larger the tumor size the higher rate of axillary lymph node metastasis.

Breast cancer axillary lymph node metastasis rate was $25.7 \%$ in T1 Group, $51.8 \%$ in $\mathrm{T} 2$ group, and in $\mathrm{T} 3$ group, the axillary lymph node metastasis rate rose to $92.3 \%$. There were statistically significant differences between different groups compassion. Recently, study confirmed, monitoring the prognosis marker on breast cancer metastasis has positive correlation with the tumor size [2]. For example, there is correction between high serum concentration (more than or equal to $2 \mathrm{mu} / \mathrm{ml}$ ) ck19-2g2 and the tumor size (more than or equal to $2 \mathrm{~cm}$ ). But in the early stage of breast cancer, tumors often cannot be checked out. So, research on relative tumor marker has a great value of diagnosing breast cancer.

Reports have revealed comedo necrosis is a valuable predictive factor of breast tumor recurrence risk factors [3]. Comedo necrosis also could be seen in small breast infiltrating cancer such as ductal carcinoma in situ, which has high risk of

\section{Age}

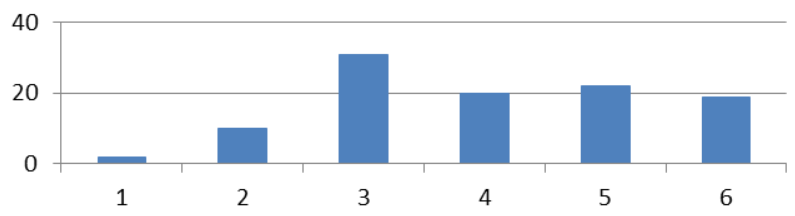

Figure 2. Age of breast cancer patients. 1: $\geq 30$ y, 2: $31-40$ y, 3: $41-50$ y, $4: 51-60$ y, 5: 61 $-70 \mathrm{y}, 6: \geq 71 \mathrm{y}$. 
axillary lymph node metastasis [4]. And the prognosis of comedo necrosis ductal carcinoma in situ was more poor than the non-comedo necrosis ductal carcinoma in situ [5] [6]. Within the 104 cases of breast cancer tissues, we got 9 cases of patients with carcinoma in situ. Therefore, we analyze the relationship among the comedo necrosis, stage and axillary lymph node metastasis. Our results shown there was no statistically significant difference between comedo necrosis and lymph node metastasis (Table 2), but there still was 39/103 of lymph node metastasis cases with comedo necrosis, the ratio was higher than no lymph node metastasis cases (21/103).

Then we analyzed comedo necrosis combined with lymph node metastasis cases in different breast cancer stage. In stage I there were $13.3 \%$ cases had comedo necrosis combined with lymph node metastasis, while in stage II and III, the ratio was $34.4 \%$ and $45.8 \%$ respectively (Table 3 ).

Data revealed that perhaps higher tumor stage in ductal breast cancer has higher ratio of comedo necrosis lesions and lymph node metastasis, however, there is no significant difference between the statistical results, which is most likely due to the number of limited cases.

Some scholars believe that cell mitoses represent the biological behavior of the tumor and can make greater value and clinical significance [7]. With the application of computer automatic image analysis technology in the detection of prognostic markers in breast cancer [8] [9] [10] [11] [12], the counting method of nuclear fission image now becomes more and more accurately. In this study, we watched the relationship between number of cell mitoses and axillary lymph node metastasis. Data shown that the group ( $\geq 10$ cell mitoses) showed significantly higher patient with axillary lymph node metastasis compared to the group $(0$ - 9 cell mitoses $)(P=0.0100)$.

Every 100 thousand women in the United States from 25 to 39 years of age, the incidence of breast cancer increased from $1.53 \%$ in 2009 to $2.90 \%$ in 1976 [13]. In recent years, the incidence of female breast cancer in China is also rising, and the disease incidence showed younger trend. Lymph node metastasis may reflect the biologic behavior of the tumor, to analysis the pathological features of breast cancer, and to explore the related factors of axillary lymph node metastasis, have important significance on the treatment and prognosis of breast cancer. If breast cancer recurrence, the main causes of death were axillary lymph node metastases [14]. The scope and number of the lymph node dissection can affect the treatment and prognosis of patients [15]. Through initial analysis of breast

Table 3. The relationship between the number of cell mitoses and axillary lymph nodes metastasis.

\begin{tabular}{cccc}
\hline number of cell mitoses & $\mathrm{n}$ & lymph node $(+)$ & lymph node $(-)$ \\
\hline $0-9$ & 54 & $19(35.2 \%)$ & $35(64.8 \%)$ \\
$\geq 10$ & 50 & $31(62.0 \%)$ & $19(38.0 \%)$
\end{tabular}

$\mathrm{n}$ : number of cases. The number of patients with axillary lymph node metastasis in group II was more than that of group I; the difference was statistically significant $(P=0.0139 ; P<0.05)$. 
cancer pathological type, we believe that breast cancer with axillary lymph node metastasis is closely related to the tumor size, and the number of cell mitoses is closely related to axillary lymph node metastasis too. Therefore, more attention should be paid to comedo necrosis during clinical process.

\section{Acknowledgements}

This study was supported by Shanghai Pudong Science and Technology Commission, China (Grant No.: PKJ2014-Y23), Shanghai University of Medicine \& Health Sciences (Project of Hundred people Library: A4-2601-17-311001 and The Seed fund program: SFP-17-21-05-02).

\section{References}

[1] Rosa Mendoza, E.S., Moreno, E. and Caguioa, P.B. (2013) Predictors of Early Distant Metastasis in Women with Breast Cancer. J Cancer Res Clin Oncol, 139, 645-652. https://doi.org/10.1007/s00432-012-1367-Z

[2] Kong, Y., Wang, J., Liu, W., Chen, Q., Yang, J., Wei, W., Wu, M., Yang, L., Xie, X., Lv, N., Guo, J., Li, L., Gao, J., Xie, X. and Dai, S. (2013) Cytokeratin19-2g2, a Novel Fragment of Cytokeratin19 in Serum, Indicating a More Invasive Behavior and Worse Prognosis in Breast Cancer Patients. PLoS ONE, 8, e57092.

https://doi.org/10.1371/journal.pone.0057092

[3] Fisher, E.R., Land, S.R., Saad, R.S., Fisher, B., Wickerham, D.L., Wang, M., Costantino, J.P. and Wolmark, N. (2007) Pathologic Variables Predictive of Breast Events in Patients with Ductal Carcinoma in Situ. Am J Clin Pathol, 128, 86-91. https://doi.org/10.1309/WH9LA543NR76Y29J

[4] Lee, J.H., Suh, Y.J., Shim, B.Y. and Kim, S.H. (2011) The Incidence and Predictor of Lymph Node Metastasis for Patients with T1mi Breast Cancer Who Underwent Axillary Dissection and Breast Irradiation: An Institutional Analysis. Jpn J Clin Oncol, 41, 1162-1167. https://doi.org/10.1093/jjco/hyr128

[5] Stackievicz, R., Paran, H., Bernheim, J., Shapira, M., Weisenberg, N., Kaufman, T., Klein, E. and Gutman, M. (2010) Prognostic Significance of HER-2/Neu Expression in Patients with Ductal Carcinoma in Situ. Isr Med Assoc J, 12, 290-295.

[6] Shekhar, M.P., Tait, L., Pauley, R.J., Wu, G.S., Santner, S.J., Nangia-Makker, P., Shekhar, V., Nassar, H., Visscher, D.W., Heppner, G.H. and Miller, F.R. (2008) Comedo-Ductal Carcinoma in Situ: A Paradoxical Role for Programmed Cell Death. Cancer Biol Ther, 7, 1774-1782. https://doi.org/10.4161/cbt.7.11.6781

[7] Lehr, H.A., Rochat, C., Schaper, C., Nobile, A., Shanouda, S., Vijgen, S., Gauthier, A., Obermann, E., Leuba, S., Schmidt, M.C.C.R., Delaloye, J.F., Simiantonaki, N. and Schaefer, S.C. (2013) Mitotic Figure Counts Are Significantly Overestimated in Resection Specimens of Invasive Breast Carcinomas. Modern Pathology, 26, 336-342. https://doi.org/10.1038/modpathol.2012.140

[8] Sarode, V.R., Han, J.S., Morris, D.H., Peng, Y. and Rao, R. (2011) A Comparative Analysis of Biomarker Expression and Molecular Subtypes of Pure Ductal Carcinoma in Situ and Invasive Breast Carcinoma by Image Analysis: Relationship of the Subtypes with Histologic Grade, Ki67, p53 Overexpression, and DNA Ploidy. International Journal of Breast Cancer, 2011, 1-7. https://doi.org/10.4061/2011/217060

[9] Bolton, K.L., Garcia-Closas, M., Pfeiffer, R.M., Duggan, M.A., Howat, W.J., Hewitt, S.M., Yang, X.R., Cornelison, R., Anzick, S.L., Meltzer, P., Davis, S., Lenz, P., Fi- 
gueroa, J.D., Pharoah, P.D. and Sherman, M.E. (2010) Assessment of Automated Image Analysis of Breast Cancer. Tissue Microarrays for Epidemiologic Studies. Cancer Epidemiol Biomarkers, 19, 992-999. https://doi.org/10.1158/1055-9965.EPI-09-1023

[10] Mohammed, Z.M.A., McMillan, D.C., Elsberger, B., Going, J.J., Orange, C., Mallon, E., Doughty, J.C. and Edwards, J. (2012) Comparison of Visual and Automated Assessment of Ki-67 Proliferative Activity and Their Impact on Outcome in Primary Operable Invasive Ductal Breast Cancer. British Journal of Cancer, 106, 383-388. https://doi.org/10.1038/bjc.2011.569

[11] Konsti, J., Lundin, M., Joensuu, H., Lehtimäki, T., Sihto, H., Holli, K., Turpeenniemi-Hujanen, T., Kataja, V., Sailas, L., Isola, J. and Lundin, J. (2011) Development and Evaluation of a Virtual Microscopy Application for Automated Assessment of Ki-67 Expression in Breast Cancer. BMC Clinical Pathology, 11, 3-11.

https://doi.org/10.1186/1472-6890-11-3

[12] Fasanella, S., Leonardi, E., Cantaloni, C., Eccher, C., Bazzanella, I., Aldovini, D., Bragantini, E., Morelli, L., Cuorvo, L.V., Ferro, A., Gasperetti, F., Berlanda, G., Dalla, Palma, P. and Barbareschi, M. (2011) Proliferative Activity in Human Breast Cancer: Ki-67 Automated Evaluation and the Influence of Different Ki-67 Equivalent Antibodies. Diagnostic Pathology, 6, S1- S7. https://doi.org/10.1186/1746-1596-6-S1-S7

[13] Johnson, R.H., Chien, F.L. and Bleyer, A. (2013) Incidence of Breast Cancer with Distant Involvement among Women in the United States, 1976 to 2009. JAMA, 309, 800-805. https://doi.org/10.1001/jama.2013.776

[14] Fayanju, O.M., Nwaogu, I., Jeffe, D.B. and Margenthaler, J.A. (2015) Pathological Complete Response in Breast Cancer Patients Following Neoadjuvant Chemotherapy at a Comprehensive Cancer Center: The Natural History of an Elusive Prognosticator. Mol Clin Oncol, 3, 775-780. https://doi.org/10.3892/mco.2015.535

[15] Baghestani, A.R., Shahmirzalou, P., Zayeri, F., Akbari, M.E. and Hadizadeh, M. (2015) Prognostic Factors for Survival in Patients with Breast Cancer Referred to Omitted Cancer Research Center in Iran. Asian Pac J Cancer Prev, 16, 5081-5084. https://doi.org/10.7314/APJCP.2015.16.12.5081 